\title{
Philosophiques
}

\section{Pleurer et rire pour de vrai}

\section{Maurizio Ferraris}

Volume 40, numéro 1, printemps 2013

Littérature et connaissance

URI : https://id.erudit.org/iderudit/1018374ar

DOI : https://doi.org/10.7202/1018374ar

Aller au sommaire du numéro

Éditeur(s)

Société de philosophie du Québec

ISSN

0316-2923 (imprimé)

1492-1391 (numérique)

Découvrir la revue

Citer cet article

Ferraris, M. (2013). Pleurer et rire pour de vrai. Philosophiques, 40(1), 23-44. https://doi.org/10.7202/1018374ar

\section{Résumé de l'article}

L'une des réponses au paradoxe de la fiction consiste à dire que les émotions que nous éprouvons face aux oeuvres de fiction ne sont pas véritables. Mais qu'est-ce que pleurer ou rire pour de vrai ? En fait, presque toutes les formes de rire ou de larmes, et de réactions émotionnelles, sont compatibles avec la fiction, y compris celles qui sont des émotions vraies. Ce qui pose problème dans le paradoxe est la prémisse selon laquelle nos croyances au sujet de la fiction doivent être vraies ou fausses.
Ce document est protégé par la loi sur le droit d'auteur. L'utilisation des services d'Érudit (y compris la reproduction) est assujettie à sa politique d'utilisation que vous pouvez consulter en ligne.

https://apropos.erudit.org/fr/usagers/politique-dutilisation/ 


\title{
Pleurer et rire pour de vrai
}

\author{
MAURIZIO FERRARIS \\ Università degli Studi di Torino
}

\begin{abstract}
RÉSUMÉ. - L'une des réponses au paradoxe de la fiction consiste à dire que les émotions que nous éprouvons face aux œuvres de fiction ne sont pas véritables. Mais qu'est-ce que pleurer ou rire pour de vrai? En fait, presque toutes les formes de rire ou de larmes, et de réactions émotionnelles, sont compatibles avec la fiction, y compris celles qui sont des émotions vraies. Ce qui pose problème dans le paradoxe est la prémisse selon laquelle nos croyances au sujet de la fiction doivent être vraies ou fausses.
\end{abstract}

SUMMARY. - One of the answers to the paradox of fiction consists in claiming that the emotions that we feel about fictions are not genuine emotions. But what is it to laugh or to cry genuinely? In fact, almost all kinds of laughter and of cry are compatible with fictions, including genuine ones. What is wrong in the paradox of fiction is the premise according to which our beliefs about fictions have to be true or false.

Il est ici question du paradoxe de la fiction. Ce paradoxe semble chose très abstraite, lointaine ou académique, mais en fait il concerne une part importante de notre vie, celle que nous consacrons à des choses comme aller au cinéma, lire des romans, regarder une série télévisée - toutes choses destinées à nous amuser, mais pas toujours, parce que parfois on finit par en pleurer. Le paradoxe est le suivant: I) Maurizio Ferraris est attristé par la fin tragique d'Anna Karénine, et Maurizio Ferraris sait parfaitement qu'Anna Karénine est une entité fictive; 2) Croire à l'existence de ce qui nous rend triste est une condition nécessaire pour avoir des émotions; 3) Maurizio Ferraris ne croit pas à l'existence d'une entité fictive; donc Maurizio Ferraris n'est pas vraiment ému. Il est une sorte d'hypocrite, plus précisément un autohypocrite qui se dupe lui-même et ose appeler cette situation peu édifiante "plaisir esthétique» et peut-être aussi «enrichissement culturel».

Le problème est sérieux et concerne aussi bien le «pleurer » que le "vraiment». Que signifie "pleurer vraiment» ? Ou plutôt «pleurer sérieusement», le «pleurer à juste titre» dont sont menacés les enfants lorsqu'ils font des caprices? "Si tu ne t'arrêtes pas je te donne une fessée, et alors tu pleureras à juste titre ». La menace maternelle laisse entendre qu'on peut avoir des pleurs feints, et que l'enfant est hypocrite comme moi quand je m'émeus pour Anna Karénine.

L'exemple de la mère qui menace son enfant de "pleurer à juste titre » (et donc insinue qu'il ne pleure pas vraiment) suggère à quel point il est compliqué de comprendre ce que signifie «vraiment » — «vraiment ", quand on fait allusion aux pleurs ou à d'autres sentiments (et en particulier au rire).

Dans le cas de la mère, "vraiment" signifie plus ou moins: "Petite canaille, si tu ne t'arrêtes pas je te ferai pleurer pour des douleurs du corps 
et de l'âme, celles-là mêmes au nom desquelles tu es en train de me casser les pieds. " La mère applique un principe drastiquement réductionniste: si on ne pleure pas pour des douleurs physiques, alors on ne pleure pas vraiment. Il faut que ce soient des douleurs physiques; le simple pleur physique, mais sans douleur physique, par exemple quand on pèle des oignons, n'est pas valable.

En fait, on pourrait carrément imaginer la mère transformée en marâtre cruelle, qui découvre le fils pleurant à la cuisine et qui, par jeu (mieux, «pour rire») a pelé des oignons pour le faire pleurer «vraiment ", afin qu'il pleure à juste titre. Du point de vue logique elle aurait raison, du point de vue moral elle serait détestable, et même une excellente candidate à l'infanticide.

Supposons maintenant que notre marâtre meure accidentellement. On l'enterre, et le fils suit le cercueil. Supposons qu'il pleure: pleurerait-il à juste titre? Il est difficile de le dire, du moins si l'on s'en tient aux standards de la défunte. En fait, le fils ne pleurerait pas à cause d'une souffrance physique, mais à cause d'une de ces douleurs de l'âme qui, pour la défunte, ne pouvaient pas être considérées comme des causes légitimes de vrais pleurs.

Qui plus est, celui qui penserait que la douleur de l'âme peut être la cause de pleurs légitimes aurait de très bonnes raisons de se demander quelle douleur de l'âme peut ressentir le fils lors du décès de sa mère. Au fond, cette mère n'a été qu'une horrible castratrice insensible à ses douleurs. S'il pleure, le fils ne pleure pas à juste titre. C'est un hypocrite. Et s'il pleure sans hypocrisie, il faudrait se demander s'il pleure vraiment, s'il pleure à juste titre, parce qu'il n'est pas juste de pleurer une mère comme ça: en pleurant, le fils s'abandonne simplement au syndrome de Stockholm.

Laissons le fils de la marâtre, et passons au père, c'est-à-dire au conjoint de la défunte. Lui aussi accompagne le cercueil, et pleure. Pleure-t-il vraiment ? Là aussi, les raisons de douter sont nombreuses, et on a autant de raisons de dire que le conjoint ne pleure pas vraiment et ne pleure pas à juste titre. Beaucoup de gens, à le voir pleurer, penseraient que c'est de la blague, que ce ne sont pas de vrais pleurs. Après tout, il s'est libéré d'une harpie ou tout au moins d'une maniaque de la sincérité, qui poussait son impitoyable recherche de l'absolu sentimental jusqu'à tourmenter son fils en l'empêchant de peler ses oignons et de pleurer pour des motifs insuffisants. La tempête est passée, peut-être que ce pauvre homme un peu lâche et un peu complice pleure maintenant, mais il feint de le faire et ne pleure pas vraiment. Ou peutêtre pleure-t-il non pas par douleur, mais plutôt hystériquement, par compulsion, par une espèce d'acte libérateur. Donc, là encore, il ne pleure pas vraiment. Mais imaginons qu'il pleure exactement parce que la mort de cette virago lui fait de la peine, imaginons qu'il pleure sincèrement. Il aurait encore des raisons (les gens sont méchants) de douter du fait que ses pleurs soient vrais: c'est juste que le pauvre homme, en plus d'être lâche et complice, est également bête et ne s'est pas rendu compte que sa femme était un 
monstre. C'est pourquoi il ne pleure pas à juste titre, parce qu'il devrait plutôt rire, ou mieux, remercier le Ciel.

Pleurer vraiment est chose difficile, comme le montrent ces exemples un peu gothiques. Pourquoi? La raison est simple: il n'est pas facile de dire ce que c'est que pleurer à juste titre. Mais les raisons des autres, les raisons qui les font pleurer, sont difficiles à vérifier et encore plus à partager. Il semble toujours que les autres pleurent sans motif, bref qu'ils ne sachent pas pourquoi ils pleurent, mais qu'ils pleurent pour des raisons futiles, pour des motifs insuffisants à justifier leurs pleurs (ou leur rire, surtout si les autres rient de nous). S'agissant de la difficulté de vérifier les raisons d'autrui, il n'est pas nécessaire d'être un existentialiste pour comprendre que les autres, surtout dans leurs sentiments, sont impénétrables, ou du moins peuvent l'être, ou qu'il est commode de penser qu'il en est ainsi. Nous voyons quelqu'un pleurer, il nous semble qu'il n'a pas de motifs suffisants pour le faire, et nous désapprouvons: "Qu'est-ce qu'il a à pleurer, celui-là ? " En vain, l'autre, la voix cassée par les sanglots, nous expliquera que l'Autre est complètement transcendant, que nous ne réussirons jamais à nous mettre à sa place, que l'individu est ineffable, et qu'en dépit de tout cela il a de bonnes raisons de pleurer. Nous continuerons à penser qu'il n'est pas en train de pleurer à bon escient, qu'il pleurniche, etc., et qu'il continuera à pleurer, et de ce fait pleurera encore plus, parce qu'il se sentira seul dans sa douleur.

Il est difficile de pleurer vraiment, à la fois parce qu'autrui est inconnaissable - du moins si l'on en croit les existentialistes - et surtout parce que, dans le pleur et le rire à bon escient se cache un puissant élément normatif. On peut pleurer ou rire seulement à certaines conditions, et si on pleure ou rit sans satisfaire à ces conditions, alors il est exclu que l'on pleure ou rie vraiment, et il faut prendre des mesures comme «pleurer à juste titre» ou «rira bien qui rira le dernier».

La normativité du pleur et du rire devient à ce point une espèce de règle d'humanité. Dans l'abstrait, c'est vrai: après tout, il n'y a que les hommes qui pleurent ou qui rient, les animaux tout au plus montrent les dents, c'est une autre paire de manches. Mais dans la vie réelle il faut considérer les raisons: "on ne pleure pas sur ceci », " on ne rit pas de cela». Mais quelles sont les choses sur lesquelles on peut pleurer ou rire? Cela se décide en privé. Et la censure intervient de deux façons. D'abord, on peut nous dire que nous rions ou pleurons pour des broutilles. Ensuite on peut nous dire que les raisons pour lesquelles nous rions ou pleurons sont inadaptées, qu'on ne rit ni ne pleure pour des choses comme ça: il est répugnant de rire d'un génocide, mais en pleurer est aussi trop peu, ce n'est pas pleurer qui convient. Apparemment, on peut rire ou pleurer seulement de choses intermédiaires, mais qu'est-ce qu'une chose intermédiaire?

Pleurer vraiment est difficile. S'il en est ainsi, il serait très difficile de prétendre que celui qui pleure sur le sort d'Anna Karénine pleure «moins vraiment ", que celui qui pleure pour sa femme. Je me rends compte que le cas 
du mari qui pleure la mort de sa femme alors qu'elle était une virago semble tiré par les cheveux. On pourrait dire: "Ça suffit! Celui qui pleure au sujet de sa propre femme pleure vraiment; celui qui pleure pour Anna Karénine ne pleure pas vraiment. Inutile de compliquer les choses!» Mais pleurer au sujet de sa femme, dans des conditions normales, peut conduire à l'insincérité. En effet, substituons "X pleure sa première femme" par «X pleure sa femme ». On a des raisons de douter de la sincérité des pleurs. Après tout, s'ils ont divorcé, c'est qu'ils le voulaient, et alors pourquoi pleurer? Souvent, en effet, le divorcé ne pleure pas. Pensez aux enterrements, où il y a plus souvent des veuves que des veufs parce que les hommes meurent plus tôt: la deuxième femme pleure, la première ne pleure pas, ou rarement. Veut-on que $\mathrm{X}$, l'ex-conjoint de notre exemple, si pour quelque raison sa première femme le précède dans l'ultime voyage, se comporte autrement? Et si au contraire il pleure, il y a de fortes raisons de le considérer comme un hypocrite. Pourquoi serait-il en train de jouer la comédie? Pour ses enfants? Ou pour taquiner sa deuxième femme? Si l'on est dans le second cas, cela montre que pleurer sa propre femme est toujours insincère: $\mathrm{X}$ en effet pleure (non sincèrement) la première femme pour taquiner la deuxième avec laquelle il s'est disputé; si la deuxième mourait, il n'y a pas de doute que les pleurs de X qui n'a pas hésité à simuler — seraient insincères.

Karénine, le mari d'Anna Karénine, n'a pas versé une larme lorsqu'elle s'est jetée sous le train. Dans ce cas, on ne peut jamais le dire, parce que cet imbécile de Charles Bovary, inconsolable, a au contraire pleuré Emma. Mais il est bête, et ce sont des personnages de fiction. Cependant, prêtons attention à un fait qui s'est produit il y a quelques années, la mort de Diana Spencer, Lady Di. Inexplicablement selon moi, mais de manière empiriquement irréfutable, des millions de personnes dans le monde ont pleuré sa mort, ils ont apporté des fleurs dans le tunnel de l'Alma, ils ont suivi les enterrements les plus chanceux en «live ", les autres à la télévision. Et il n'est pas difficile d'imaginer un quelconque détenu dans le corridor de la mort des quartiers de sécurité de la prison d'Ellis, au Texas, pleurer comme un veau en regardant l'enterrement de Lady Di. Mais si un détenu dans le corridor de la mort pleure vraiment Lady $\mathrm{Di}$, pourquoi ne pourrait-il pleurer vraiment pour Anna Karénine? Le vrai problème, au bout du compte, est de comprendre pourquoi il n'a pas versé une larme lorsqu'il commettait le massacre qui l'a conduit vers le corridor de la mort.

Tout ce que cela montre, c'est qu'il y a plusieurs façons de pleurer. Passons-les en revue. La première façon de pleurer est ce qui se passe quand on pèle des oignons. Je propose de l'appeler "pleur phénoménologique» parce qu'il a l'apparence extérieure des pleurs mais n'a aucun équivalent interne ou physique. En effet, quand on pleure à cause des oignons on n'est pas triste, on est tout au plus agacé. Mais on peut bien imaginer quelqu'un qui pèle les oignons pour faire un risotto qu'il offrira à Monica Bellucci. Il pleure, à coup sûr, mais il est aussi heureux et plein d'espoir. Toutefois, il y a des cas pro- 
blématiques, par exemple, si l'on imagine notre peleur d'oignons, solitaire et abandonné, se souvenant du temps où il déjeunait avec Monica Belluci. L'abandonné pleure; mais, il peut se demander: "Suis-je en train de pleurer sur mon passé? Ou suis-je simplement triste, et mes pleurs sont-ils simplement phénoménologiques, c'est-à-dire causés par les oignons que je suis en train de couper?" Si le sommeil obtenu avec un somnifère est clairement un vrai sommeil, et pas seulement un sommeil apparent, il semble que le problème des pleurs phénoménologiques ressemble à celui du rire provoqué par le chatouillement, qui a tous les effets d'un rire phénoménologique. Souvent, celui qu'on chatouille n'a aucune envie de rire, et pourtant il rit aux éclats, et cela lui fait même mal.

Considérons à présent les pleurs provoqués par le vin, quand on a «le vin triste ». S'agit-il d'une autre version des pleurs (et du rire) phénoménologiques? Nullement. Je proposerai, pour le vin triste, de parler de "pleurs chimiques », et de distinguer ceux-ci soigneusement des pleurs phénoménologiques. La raison en est très simple. Celui qui pleure en pelant les oignons peut être parfaitement gai, ou indifférent. Celui qui pleure sous l'effet du vin, au contraire, n'est pas du tout gai, mais vraiment triste: c'est donc qu'il pleure vraiment.

Une objection possible à cet argument pourrait être la suivante. Celui qui pleure (ou rit) chimiquement ne pleure pas vraiment parce que, bien qu'il ait l'impression d'être triste ou gai, en réalité il n'y a rien dans le monde extérieur qui le rende triste ou gai, si bien que sa tristesse n'est pas une vraie tristesse, mais une espèce de dépression, tout comme il n'éprouve pas de vrai bonheur, mais plutôt une sorte d'euphorie.

C'est confirmé par la facilité avec laquelle le langage ordinaire fait la distinction entre d'un côté l'euphorie et la dépression, en tant qu'apparences, et de l'autre le bonheur et la tristesse en tant que sentiments authentiques. Les pleurs et le rire chimiques ne sont pas très différents des pleurs et du rire phénoménologiques. Malgré cela, je suis porté à croire que les pleurs chimiques sont plus authentiques que les pleurs phénoménologiques. Non pas parce que nous avons des larmes qui ne sont pas causées par la tristesse, mais aussi parce que si l'on commence à chercher la petite bête sur la réalité des objets qui provoquent nos sentiments on se trouvera obligé de penser que celui qui, après avoir abusé du vin, est en proie à une attaque de delirium tremens, voit des images terribles et a une horrible peur, et n'a pas vraiment peur parce que les scolopendres vertes qui montent sur son corps sont de simples hallucinations.

Il reste que ce pleur vineux et chimique n'a pas de causes externes mais seulement des causes internes; il n'est pas convaincant. Le paradoxe de la fiction dont nous étions partis ne tient plus trop. On peut toutefois se demander légitimement s'il faut que la cause externe soit présente à tout prix. Je m'explique: on peut se rappeler une chose ou une personne qui n'est plus, et pour cela se mettre à pleurer. Par exemple cela se passe chez Proust. Où est 
partie Albertine? Elle a disparu. On commence à pleurer. Puis on s'habitue au manque, une semaine passe, le coiffeur vient à la maison, et on se rappelle qu'une semaine avant Albertine était là, et on se met à pleurer de nouveau. Proust appelle ce phénomène "intermittence du cœur ", et c'était au départ le titre qu'il voulait donner à la Recherche.

De même Montaigne nous raconte que, pendant son voyage en Italie, près de Bolzano, lui est revenu le souvenir de son ami, Étienne de la Boétie, et qu'il s'est mis à pleurer. La scène doit avoir étonné un peu ses proches, comme les pleurs de Proust auraient étonné le coiffeur, mais il aurait suffi que Proust et Montaigne aient expliqué: "Je me suis souvenu d'une personne chère disparue »; tous auraient compris et n'auraient pas objecté que leur ami n'était pas en train de pleurer vraiment parce que l'objet de leurs pleurs n'était plus présent.

La vie psychique est une chose compliquée, la mémoire est difficile à maîtriser, la nostalgie est canaille et, comme le disaient Freud et Breuer dans les Études sur l'hystérie, "l'hystérique souffre de réminiscences". Bref, celui qui pleure du fait d'un souvenir triste pleure vraiment, exactement comme rit vraiment celui qui, à un enterrement, éclate de rire pour s'être rappelé une blague.

Donc, celui qui pleure en se souvenant pleure vraiment. Mais qu'en est-il du pleur dans le rêve? On pourrait dire que c'est plus ou moins comme pleurer sur un souvenir, mais ce n'est pas exactement ça. Se rappeler quelque chose alors qu'on est éveillé, qu'on a une mémoire consciente, c'est se souvenir vraiment. Alors que voir des images dans un rêve est toute autre chose, bien que ce ne soit pas exactement nous qui voyions ces images, mais plutôt notre inconscient, et on peut douter que cet inconscient coïncide avec notre moi conscient. Ces images sont désordonnées: le sommeil et la veille, disait Schopenhauer, sont des pages du même livre, sauf que dans le sommeil elles coulent au hasard.

Pourtant, lorsqu'on a pleuré en rêve, n'a-t-on pas l'impression qu'on a pleuré vraiment? Dans certains cas, c'est vraiment intense, et après certains rêves la réalité apparaît vraiment peu de chose. Si les choses se passent dans la fiction comme dans le rêve, comment peut-on nier que celui qui pleure en lisant la triste fin d'Anna Karénine pleure vraiment? Après tout, les pages du livre, à la différence du rêve, sont parfaitement ordonnées, elles sont sûrement ce que nous lisons, et nous avons conscience qu'elles nous font pleurer.

Peut-on être heureux en rêve? Peut-on être effrayé en rêve? Quand peut-on prétendre que quand on pleure en rêve on pleure vraiment? Tout le monde a l'expérience, fréquente quand on est jeune, de rêves dont a du mal à se remettre, exactement comme cela se passe pour certains romans et certains films. Et c'est dans cette étrange expérience que réside selon moi un des secrets de la littérature.

Dans un petit livre, Le rêve de Benjamin, écrit peu avant sa mort, Jacques Derrida se demandait: un rêveur peut-il parler de son rêve sans se 
réveiller? Derrida répond «Non»; l'artiste répond: "Peut-être, parfois». En définitive, ce que nous découvrons ici est ce «Peut-être, parfois », qui pourtant ne concerne pas seulement l'artiste, mais nous-mêmes, lorsque nous lisons des romans, allons au cinéma, rêvons ou nous rappelons certaines choses.

Walter Benjamin a en commun avec Socrate une mort volontaire, à cette nuance près que, dans le cas de Benjamin, cette mort est basée sur une équivoque. Benjamin était réfugié en France parce que juif et communiste, mais l'Allemagne de Hitler envahit la France, et le I4 juin les Allemands sont à Paris. Benjamin fuit vers l'Espagne. Le 25 septembre, il est à la frontière avec d'autres, mais les autorités espagnoles ne les laissent pas entrer. Bouleversé, Benjamin se suicide dans la nuit en ingérant une grande quantité de morphine. Le matin suivant, les autorités changent d'avis et accordent le passage aux fugitifs. À cette nuance près que Benjamin est désormais mort.

J'ai lu d'autres versions de cette histoire. Il semble qu'en réalité les réfugiés étaient déjà en Espagne, et que Benjamin craignait d'être arrêté par la police espagnole et confié aux Allemands, mais cela a peu importance, étant donné que de toute façon ses compagnons de fuite se sont sauvés et ont enterré son corps. Comble d'ironie dans cette tragédie des équivoques, ils ont loué sa sépulture seulement pour cinq ans, et en I945 le corps de Benjamin a été jeté on ne sait où.

Quelle que soit la bonne version, Benjamin s'est tué par erreur, parce qu'il craignait d'être arrêté et en dernière instance parce qu'il craignait de mourir. Et exactement parce qu'il craignait de mourir, peut-être par peur d'avoir peur, il a décidé de se tuer. Le point est que Benjamin s'est tué par erreur, craignant quelque chose qui n'est pas advenu. Mais qui oserait soutenir que Benjamin ne s'est pas tué vraiment? Prenons garde: ceux qui affirment que l'homme qui pleure la mort d'Anna Karénine ne pleure pas vraiment parce que cette mort n'existe que dans un roman ne disent pas autre chose que celui qui poserait que Benjamin n'est pas mort vraiment.

On peut se tuer par erreur, comme Benjamin, exactement comme on peut pleurer par erreur, et bien sûr rire par erreur («Rira bien qui rira le dernier»). Mais dans tous ces cas, on se tue, on pleure et on rit vraiment. On s'étonnera aussi qu'on puisse aussi simplement mourir par erreur. Personne ne contestera que celui qui meurt par erreur meurt vraiment. Pour être fidèle au thème de la vérité dans la littérature, prenons un exemple chez Simenon, Maigret et l'affaire Saint-Fiacre. La vieille comtesse lit dans son missel une lettre qui accuse son fils; l'accusation est fausse, la comtesse ne le sait pas et meurt sur le coup d'un infarctus. Qui nierait qu'elle soit morte vraiment? Si l'on me dit que mon parent est une fripouille, et que je ris ou pleure pour cette raison, laquelle se révèle ensuite infondée, mon rire ou mes pleurs ne sont-ils pas vrais?

Certains, comme Proust, vont nettement plus loin et inversent les rapports entre l'art et la vie. Proust soutient que lorsqu'il s'agit d'écrire on est 
attentif, méticuleux, on mesure ses mots et la vérité de ce que l'on écrit, alors que quand il s'agit de vivre on agit au hasard et on se tue pour des mensonges. Il pensait à Swann et à sa longue et malheureuse histoire d'amour avec Odette, et qui à la fin, après qu'il l'a épousée et qu'une fille est née d'elle, conclut qu'il a ruiné sa vie, son talent, sa santé et son patrimoine pour une femme qui n'était pas même son genre.

Aux récents Jeux olympiques, nous avons vu un spectacle presque sériel, celui des athlètes qui pleuraient. Un tel pleurait de tristesse, un autre parce qu'il avait perdu, parce qu'il s'était blessé au pied, etc. Pour rester dans le thème "rire ou pleurer vraiment ", je crois que ces perdants malchanceux et blessés n'auraient pas du tout apprécié des consolations du type «c'est seulement un jeu ». Ils auraient continué à pleurer vraiment, et si quelqu'un avait objecté que leurs pleurs ne sont pas de vrais pleurs parce que les Olympiades sont seulement un jeu, ils se seraient arrêtés de pleurer et lui auraient tapé dessus, pour pleurer de nouveau en toute légitimité. Pourtant, il y avait une grande quantité d'athlètes qui pleuraient parce que tout se passait rondement et qu'ils avaient gagné. Maintenant, imaginons que le même importun qui avait cherché à consoler les perdants en argumentant "cen'est-qu'un-jeu » ait cherché à consoler les gagnants en arguant que non seulement c'est un jeu mais qu'en plus ils avaient gagné et par conséquent ne devaient pas pleurer mais plutôt rire, bien qu'avec modération, précisément parce qu'il ne s'agit que d'un jeu. Ce fâcheux n'aurait pas été envoyé au diable, mais simplement été traité d'idiot: les athlètes lui auraient dit qu'ils n'avaient nul besoin d'être consolés, justement parce qu'ils pleuraient de bonheur et non pas de tristesse. Faut-il conclure que le pleur de bonheur n'est pas vraiment un pleur, que c'est un pleur phénoménologique, comme pour les oignons? Non pas parce qu'on pleure de bonheur, non pas par stimulation des yeux, mais en raison d'une passion de l'âme, exactement comme lorsqu'on pleure de tristesse. Celui qui pleure de bonheur n'a pas besoin d'être consolé, mais il pleure vraiment, bien que ce soit pour ainsi dire de manière atypique, comme lorsqu'il fait soleil et qu'il pleut.

Récapitulons les variétés de pleurs rencontrées jusqu'ici: I) Pleurer sur les oignons, le pleur phénoménologique; 2) Pleurer sous l'effet du vin, c'està-dire pleurer chimiquement ; 3 ) Pleurer sur un souvenir, c'est-à-dire pleurer consciemment, mais sans objet présent; 4) Pleurer dans un rêve, c'est-à-dire pleurer inconsciemment, mais sans objet présent; 5) Pleurer par erreur, c'està-dire pleurer à cause d'objets qui se révèlent faux; 6) Pleurer de bonheur, c'est-à-dire pleurer sans tristesse. Il semble que la vraie différence, la différence essentielle, se situe entre le pleur phénoménologique, le pleur (I), et tous les autres pleurs. En effet, lorsque je dis «les oignons me font pleurer ", j'utilise le syntagme «me font» dans un sens très différent que dans la phrase «les souffrances de l'humanité me font pleurer». Il y a une différence de direction pour ainsi dire. Dans le pleur phénoménologique, la direction est des oignons aux yeux, qui commencent à pleurer; dans tous les autres pleurs, la 
direction est de l'esprit aux yeux: nous avons certains sentiments, et commençons à pleurer.

Ces sentiments ou états d'âme pourraient aussi être conditionnés chimiquement, comme dans le cas de la cuite triste, ou bien naître de choses présentes, ou encore dériver de causes trompeuses, et le sentiment ne serait pas nécessairement celui de tristesse - il reste que la direction est toujours celle-ci: du monde à l'esprit, de l'esprit aux yeux, et de là aux larmes. Alors que dans le cas des oignons la direction est du monde aux yeux, qui commencent à larmoyer. Dans le cas du pleur induit par les oignons, le raifort ou choses du même genre, il serait plus correct de parler de «larmoiement»: comme quand on a un moucheron dans l'œil.

Avant d'aborder la question des sentiments vrais et des sentiments faux, je voudrais suggérer une très petite observation, préoccupante. Peut-être que la thèse selon laquelle pour avoir un vrai sentiment il faut avoir un objet vrai (sur laquelle s'appuie le paradoxe de la fiction) n'a pas de raison d'être. Les contre-exemples sont nombreux, et nous les avons vus. En voici un autre un peu indécent. Il serait à tout le moins bizarre de soutenir que celui qui s'excite à la vue d'un film pornographique ne s'excite pas vraiment. Les scientifiques ont expliqué que certains neurones, les «neurones miroir», ont la caractéristique de décharger aussi bien dans le cas d'actions accomplies par nous que lorsqu'on regarde les autres accomplir ces mêmes actions. Or s'il faut donner crédit à la règle selon laquelle pour avoir de vrais sentiments il est nécessaire que les objets de ces sentiments soient véridiques, il faut en inférer qu'il y a une différence essentielle entre le voyeurisme - si l'on peut dire - «en prise directe», et le visionnement de films pornographiques, alors que le sens commun nous dit, très justement, que c'est plus ou moins la même chose. Et l'argument selon lequel, dans un film pornographique, les acteurs sont vrais n'est pas valable. On peut imaginer par exemple un roman pornographique: le statut de réalité des personnages est absolument identique à celui d'Anna Karénine, ou d'Emma Bovary, à cette nuance près que, au lieu de dire seulement «elle s'abandonnait », comme le fait Flaubert pour raconter la rencontre entre son hérö̈ne et Léon, le porno-narrateur entre dans les détails. Il semblerait étrangement sélectif de soutenir qu'on ne peut pas pleurer vraiment pour Anna ou pour Emma, alors qu'on peut s'exciter vraiment pour la version pornographique de leur histoire.

Je crois qu'on peut désormais dire, sans crainte d'être démenti: celui qui pleure pour Anna Karénine pleure vraiment. Et par conséquent le paradoxe de la fiction, selon lequel celui qui éprouve des sentiments pour des objets fictifs n'éprouve pas vraiment ces sentiments n'est pas un paradoxe, parce qu'il est seulement apparent. Le vrai paradoxe auquel nous avions affaire en était un autre, celui de la tragédie. Le paradoxe de la tragédie est aussi vieux que la Poétique d'Aristote: pour quels motifs va-t-on chercher dans les livres ou dans le théâtre des scènes tristes, et pourquoi regarde-t-on des DVD de films d'horreur, alors que dans la vie normale on fuit ces mêmes 
scènes tristes, tragiques ou horribles? Il est intéressant de constater que, bien que le paradoxe de la fiction rende problématique que l'on puisse s'émouvoir de malheurs imaginaires, le paradoxe de la tragédie rend problématique que l'on cherche dans la fiction des choses que l'on fuit dans la réalité. La réponse serait donc très simple: où est le problème?

Je m'émeus de malheurs ou d'horreurs dans les œuvres artistiques parce que ces malheurs et horreurs sont bien racontés (sinon, en effet, je ne m'émouvrais pas mais m'ennuierais, ou même rirais). Ce qui m'importe n'est pas le fait que l'événement triste ou tragique soit vrai. Au contraire, s'il était vrai je ne pourrais pas éprouver de plaisir esthétique. C’est pourquoi nous demandons: si les choses étaient vraies, pleurerait-on mieux? Rien n'est moins certain. On peut le vérifier facilement. Je suis au théâtre et regarde Hamlet. À un certain point, emportée par les événements et exaspérée par Hamlet qui l'exhorte obsessionnellement à aller au couvent, Ophélie se noie. Nous sommes tous émus du malheur de la jeune fille, apprécions la façon dont la scénographie réussit à reproduire le petit lac dans le théâtre, la façon dont le réalisateur a représenté la scène, et la manière dont l'actrice exprime les sentiments d'Ophélie. À présent, imaginons que soit tout vrai. Le lac est vrai, et l'actrice se noie vraiment. Si nous venons à le savoir à la fin de la représentation, tout l'enchantement disparait, nous sommes dominés par le dégoût et réclamons que la justice punisse les responsables de cette initiative inhumaine; nous nous sentons trompés (alors que face à une noyade feinte tout le monde est satisfait). Si au contraire on apprend que tout est vrai quand l'action se déroule, on se jette dans le lac pour sauver la malheureuse. Curieux, n'est-ce pas? Si tout est feint, on pleure vraiment et on se félicite de son noble sentiment; si c'est vrai, on hurle, on enrage, on dénonce, on saute à l'eau et on nage, sans quoi on se sentirait dans une «ignoble farce».

Encore une preuve du fait que, si ce qui est représenté est vrai, nous ne pleurons plus vraiment, et dans la majorité des cas nous ne pleurons pas du tout. De temps en temps il y a des films et des livres, comme Quo vadis, où sont représentés des combats de gladiateurs et où, au meilleur moment, des chrétiens se font dévorer par les lions. Très souvent ces chrétiens nous les connaissons un peu, pour avoir entr'aperçu leurs vices et leurs vertus au cours du roman ou du film, et nous sommes émus comme nous le serions de voir des gens de notre connaissance dévorés par les lions. Mais même si nous ne les connaissions pas nous serions horrifiés, et la scène des martyrs dévorés par les lions touche les cœurs les plus durs.

Il arrive aussi qu'il n'y ait pas transposition artistique, et qu'on ait affaire à un documentaire télé sur la vie quotidienne à Rome: quand on en arrive aux martyrs dévorés par les lions, on se moque des martyrs et, avec l'avancée de la conscience animaliste, on a commencé à s'inquiéter des ravages causés aux gazelles, aux léopards, aux lions, aux rhinocéros et aux éléphants. Comme les Romains sont bêtes, comme les gladiateurs sont féroces! Par rapport à Quo vadis les choses ont changé. Plutôt que de plaindre 
les martyrs, on méprise les Romains et leurs coutumes brutales. À présent, imaginons que nous nous trouvions vraiment aux jeux du cirque, là où de vrais lions mangent de vrais martyrs. Si l'on cultive des sentiments peu amicaux vis-à-vis du christianisme, ces martyrs seront méprisés, et les sentiments seront vrais malgré les signes contraires aux autres, et ils ne seront pas moins vrais que ceux qu'on aurait en voyant Quo vadis.

Notons une autre circonstance. À l'origine, les spectateurs n'étaient ni tristes ni indignés, mais ravis de voir manger les chrétiens, exaltés comme on pourrait l'être aujourd'hui pendant un match de football. Cela montre une fois encore que, si ce qui est représenté était vrai, nous n'aurions pas des sentiments plus vrais, mais des sentiments plus variés et franchement étonnants. Qui plus est, il n'est pas besoin d'aller très loin dans le temps; il suffit de penser aux corridas ou aux courses cyclistes, où l'on se croit autorisé à ignorer que ceux qui pédalent dans les côtes en déployant des efforts surhumains qui déforment leurs visages sont dopés, ou aux rencontres de boxe, où la foule s'exalte au moment où un champion tombe au sol sous un coup qui rendrait quiconque fou, aveugle ou sourd. Le plaisir est le même que celui du Colisée, et sa règle est qu'il faut que tout soit vrai. Par contre, prenons un film sur les vicissitudes existentielles d'un toréro. Ou bien imaginons un film sur la vie de Marco Pantani, ou encore pensons à tous les films de boxe. Ici, le fait que rien de ce qui est représenté n'est vrai est la condition nécessaire aux sentiments nobles, chevaleresques, émouvants, qui doivent être transmis par le film. Parce que, disons-le franchement, si vraiment Rocky Balboa prenait un poing en pleine figure et que nous voyions gicler au ralenti des liquides indiscernables (sueur? salive? matière cérébrale?) et que nous sachions que ce poing a été reçu par Sylvester Stallone, nous aurions une réaction de dégoût et non d'émotion.

Nous sommes horrifiés de voir de vrais martyrs se faire manger par de vrais lions, des gladiateurs se faire tuer vraiment, des taureaux et des coqs se faire occire vraiment. Mais ici on a affaire à une inclination perverse au mal. Celle, par exemple, qui caractérise certains films amateurs, bien nommés "Snuff Movies", où un amateur fou et criminel demande à un réalisateur encore plus fou et plus criminel que lui de faire un film dans lequel une personne, après des souffrances terribles, meurt vraiment. Mais quelles que soient ces aberrations évidentes, il faut noter que, alors que sur scène (nécessairement celle du théâtre) intervient quelque chose de violent et de réel, par exemple quand on utilise du vrai sang d'animal dans certains happenings de Hermann Nitsch, ou comme lorsqu'en 1985 la compagnie théâtrale Magazzini criminali tua un vrai cheval sur la scène (l'événement fut très justement dénoncé, mais les hommes préfèrent les ténèbres à la lumière, et derechef, en 2009, l'artiste franco-algérien Adel Abdessemed proposa des vidéos d'animaux à l'abattoir), il y a des cas où nous avons une manœuvre typique d'avant-garde, qui se donne comme but explicite d'enfreindre les règles, 


\section{Philosophiques / Printemps 2013}

notamment celui-ci: "Jamais de vrai sang sur la scène, toujours du jus de tomate."

On observera toutefois qu'à l'origine de la tragédie, et donc de toutes ces amuseries mimétiques et ritualisées, il y eut au début le sacrifice véritable d'un homme, après celui d'un bouc. Oui, mais ces origines se perdent en des époques très lointaines. Aujourd'hui, heureusement, l'homme a changé tout au moins en surface - , mutatis mutandis.

Ajoutons une dernière considération à la thèse selon laquelle si la scène représentée est vraie, nous ne prenons plus de plaisir, voire moins, ou pas du tout. L'idée est que si la vérité des sentiments éprouvés était directement proportionnelle à notre engagement existentiel dans la scène, il y aurait alors une hiérarchie du genre: un peu de plaisir pour les scènes vues au cinéma, beaucoup de plaisir pour les scènes théâtrales, un très grand plaisir pour les scènes véridiques, comme de vrais massacres ou de vraies corridas, et un plaisir illimité pour les scènes vraies dans lesquelles nous sommes nous-mêmes engagés. Ce qui voudrait dire que le plaisir le plus éminent devrait coïncider avec le fait de se trouver impliqué dans un massacre ou tout au moins dans un naufrage.

Or ce n'est pas ainsi que les choses se passent, et la sagesse des philosophes, comme celle des gens ordinaires, nous fait comprendre qu'il est doux de faire naufrage seulement si la mer est métaphorique, et qu'au contraire si la mer est entendue au sens littéral, alors il vaut mieux profiter de la scène sur le rivage. C'est ce que disait Kant, parlant du "sublime », quelque chose de supérieur au beau, quelque chose de grandiose par ses dimensions (sublime mathématique: par exemple, les pyramides) ou son énergie (sublime dynamique: par exemple les tempêtes, ouragans et tremblements de terre). Mais Kant précisait justement que, pour prendre plaisir à l'effet sublime d'une tempête sur la mer, il vaut mieux rester à terre, bien à l'abri.

C'est ainsi que le paradoxe de la tragédie se trouve expliqué, à deux millénaires de distance, par les grandes théories d'Aristote et de Hume, qui nous disent toutes deux pourquoi nous payons pour assister à des récits de morts, de deuils, d'homicides et de malheurs que nous fuyons dans la vie alors que nous ne voudrions pas pour un empire y être impliqués.

Aristote répond avec simplicité et efficacité: en regardant ces scènes qui provoquent la terreur et la pitié, nous nous purifions, mieux, plus exactement nous nous purgeons, nous prenons un laxatif (tel est le sens premier de "catharsis", le terme technique utilisé par Aristote). Ainsi, nous nous libérons de toutes les craintes liées à la mort, aux maladies, aux accidents, aux catastrophes et aux horreurs qui assaillent nos imaginations dans la vie. Peut-être, sotto voce (Aristote ne le dit pas, mais nous ne pouvons pas l'exclure), nous disons-nous que ces catastrophes concernent les autres, pas nous, que nous sommes immunisés, que nous prenons plaisir à cette toute-puissance et à cette immortalité dont les jeunes se croient dotés, qui pensent que la mort ne concerne que les autres. 
Hume ajoute un détail important: ces événements nous plaisent dans l'art autant que dans la vie parce que dans l'art tout est mis en place et au moment juste, à la différence de la vie, où les choses arrivent toujours au moment le moins opportun, où les nouvelles des malheurs nous sont infligées par des personnes qui souffrent, bavardes, maladroites dans leur expression, propre à faire perdre patience à une sainte, et de façon à nous faire passer l'envie de compatir.

Le résultat maigre mais certain de tout cela est que la vérité des événements représentés ne rend pas plus vrais les sentiments que nous éprouvons mais les transforme plutôt en d'autres sentiments nettement désagréables. Et ce qui décide du caractère agréable de la représentation n'est pas la référence, mais le montage, ajouté à la certitude du fait que ce que nous sommes en train d'observer est une fiction.

Que l'effet purgatif dépende de la qualité de la narration et de ma conscience que la scène n'est pas vraie peut se voir par une simple comparaison. Imaginons un massacre dans un film de Peckimpah: chez les passionnés, le faux carnage provoque un effet cathartique (alors qu'il ne provoque chez ceux qui n'aiment pas ce genre que de l'indifférence ou des commentaires du style «trop de violence, trop de sang»). Imaginons au contraire qu'on voie un massacre dans un documentaire sur la guerre. Il n'aura aucun effet cathartique parce que la scène n'y est pas aussi nette et soignée que dans le film, et que c'est vrai.

Cependant, on a observé que de la voir à la télévision, avec la durée et le montage appropriés, avec la saleté, les piqûres de moustiques, la dysenterie, le sommeil et la faim, a réduit notre sensibilité à la guerre. En effet, les faisceaux verts des bombes sur Bagdad ne donnent guère l'idée de l'horreur, et à dire vrai ne donnent pas même l'idée de la guerre, qui n'est pas esthétisée (comme dans les batailles de Barry Lyndon, de la Croix de fer ou d'Apocalypse Now), mais anesthésiée.

Il est difficile de nier que l'on s'émeut aussi en écoutant certaines musiques (elles ont été conçues dans certains cas exactement à cette fin). Il y a des cas, comme celui de l'individu qui éprouve un frisson en écoutant la Marseillaise, dans lesquels il est vraiment difficile de dire quel est l'objet réel dans le monde auquel la Marseillaise fait référence et qui nous émouvrait. Mais pourquoi peut-on être ému par la Marseillaise de bonne foi, alors que si l'on s'émeut d'Anna Karénine on est hypocrite ou naif ? Inversement, si l'on voulait faire respecter la condition, il faudrait conclure que De Gaulle qui défile sur les Champs Élysées en août I944, dans Paris libéré, ne s'émeut pas du tout et fait semblant? Je ne le croirais pas, même si on me payait un million, et je continue à m'émouvoir tranquillement en écoutant la Marseillaise et Casablanca.

Jusqu'à présent, nous avons parlé du paradoxe de la fiction, en montrant qu'il est artificiel, c'est-à-dire qu'il n'y a rien de paradoxal dans le fait que l'on s'émeuve vraiment face à un événement fictif. Puis, nous avons 
discuté le paradoxe de la tragédie en montrant comment il est possible de savourer dans l'art des choses que nous fuirions dans la vie: à condition (autre preuve que le paradoxe de la fiction est un faux paradoxe) que la représentation soit fiction et pas réalité. Il resterait à traiter du paradoxe de la comédie. Mais il n'y a pas de paradoxe dans ce cas, pour au moins deux raisons.

La première est que, de la Poétique d'Aristote, il nous reste le livre sur la tragédie, mais malheureusement pas celui sur la comédie. La seconde est qu'il est douteux que, dans la comédie, Aristote ait trouvé beaucoup de paradoxes. Comme la comédie concerne des faits ridicules qui arrivent à des personnes pires que nous (c'est la définition d'Aristote), le paradoxe aurait été: pourquoi rions-nous d'événements que nous tournons en ridicule (ou censurons) dans la vie? C'est pourtant un paradoxe, pour ainsi dire, à l'eau de rose: au fond, entre rire et tourner en ridicule, la marge est étroite, et dans la représentation on a seulement à y gagner. Il peut aussi être amusant de voir un film avec des tamarri ${ }^{1}$, mais je ne voudrais pas les avoir comme voisins de parasol, ou être assis à la table à côté dans une pizzeria. Entre un vrai tamarro et un film sur les tamarri, personne n'hésiterait à choisir le film, à moins que (et malheureusement il fallait aussi que cela arrive) le film n'ait été fait par un tamarro. Dans ce cas, les faux tamarri nous feront regretter les vrais, à la seule exception du réalisateur.

Mais on ne comprend pas pourquoi on devrait aller au cinéma chercher ces tamarri qu'on fuit à la plage. À cela on peut répliquer deux choses. En premier lieu on peut nourrir envers les tamarri des sentiments ambivalents, de haine, mais aussi d'amour. Après tout, les journaux populaires ne sont qu'une grande comédie de tamarri, et, en termes métaphysiques, la question sonne ainsi: celui qui consulte l'album de photos exclusives du mariage Briatore-Gregoraci ${ }^{2}$ le fait-il pour les plaindre ou parce qu'il les aime un peu? En second lieu, ce qu'est vraiment un tamarro, personne ne le sait. Et surtout personne ne sait, dans le fond de sa conscience, s'il est lui-même un tamarro ou pas. Au fond, la règle est toujours: "Ce sont tous des tamarri, sauf moi ", mais il est clair que cette règle laisse place au doute, à l'examen de conscience, au regret, à l'auto-accusation. Dans le doute, donc, rire des tamarri est une bonne stratégie pour se mettre l'esprit en paix: les tamarri ne sont pas nous, mais les autres, tous les autres.

Ce doute est terrible, et peut-être ne pouvons-nous pas tous le soutenir. C'est évidemment parce que le monde est plein de tamarri qui n'admettent

1. Personnages niais de style vulgaire-chic hantant les lieux à la mode et les plages en Italie, et caricaturés dans la série de téléréalité américaine Jersey Shore. L'expression en Italie désigne une sorte de débile ridicule plus ou moins ado et plus ou moins branché. Il est difficile de traduire en français: "connard » serait le plus approchant, peut-être «moron » en québécois, mais en y ajoutant une connotation «bling-bling» (N.d.T.).

2. Noms de deux people italiens, eux-mêmes tamarri, Flavio Briatore et Eliabetta Gregoraci (N.d.T.). 
pas en être. Archivons donc l'hyperbole, et considérons ce qui suit. Même si la limite entre le tamarro et le non-tamarro était tracée de manière nette, et si nous étions sûrs de ne pas être des tamarri, il resterait que c'est confortable, presque divertissant, de suivre des tamarri au cinéma ou dans les journaux, c'est-à-dire en restant dans notre fauteuil, sans nous mêler à eux.

En définitive, c'est le même raisonnement que nous avons développé plus haut à propos de la guerre au cinéma, qui peut être noble, glorieuse, dynamique et esthétique, alors que la vraie guerre est faite d'ennuis, de mauvais sommeil, de maux de toutes sortes, de puanteurs et de douleurs; elle nous implique directement et nous interpelle alors que nous avons autre chose en tête et aucune envie de voir des scènes de guerre. Avec les tamarri, c'est la même chose. Après avoir passé une nuit d'été à lire Montaigne, ce peut être un vrai repos que d'allumer la télé et voir Boldi et $\mathrm{De} \mathrm{Sica}^{3}$, mais j'aimerais vous voir en vacances sur le Nil avec ces deux zigotos.

Une variante élégante de la comédie, c'est-à-dire de l'anthropologie du tamarro, est la tamarrizzazione du sublime, qui consiste à montrer que dans tout ce qui est élevé, et dans l'exigence même d'être sublime, il y a quelque chose d'incoerciblement tamarro, et même plus tamarro que quoi que ce soit d'autre. On note souvent que l'essence du tamarro, comme déjà celle du bourgeois gentilhomme, est l'aspiration au sublime et à l'élévation sociale, et ce n'est pas un hasard si la chose la plus tamarro d'Italie s'appelle Billionnaire.

Mais pour démontrer qu'au Billionnaire tous sont des tamarri il ne faut vraiment pas grand-chose. Il est plus difficile de montrer que les lords anglais de l'époque victorienne et les aristocrates du faubourg Saint-Germain étaient aussi des tamarri. Pour cela, il ne suffit pas d'un mauvais métier dans le style Vanzina, il faut avoir un art consommé, celui d'Oscar Wilde et de Marcel Proust, pour faire prendre Lady Windermere et le duc de Guermantes pour des tamarri. Toutefois, pour ces sommes artistiques, l'arme du tu quoque transcendantal est toute prête: s'ils sont aussi tamarri, pourquoi as-tu passé toute ta vie à les fréquenter? Ne serais-tu pas toi aussi un tantinet tamarro?

Le tamarro véritable a pourtant ça de bon qu'il sait rire de lui-même, lorsque le lord et le duc se vexent et se vengent sans pitié si l'on se moque d'eux. Proust s'en est sorti plus ou moins, parce que sa satire est pleine d'affection, mais on ne souhaiterait à personne la fin qui fut celle de Wilde et de Capote.

La stratégie inverse à la tamarrizzazion du sublime est la sublimation du tamarro. C'est plus pratiqué. Si l'on veut écouter Patty Pravo, et si on la préfère à Luigi Nono, si l'on aime la boîte des soupes Campbell's et qu'on ne comprend rien à Picasso, une porte de sortie consiste à déclarer que l'on aime le kitsch, le Camp et le pop, et on fera une très bonne impression.

3. Comiques italiens vulgaires et niais (N.d.T.). 
Le pardon s'étend-il aussi à Vanna Marchii ${ }^{4}$ et à $\mathrm{Al} \mathrm{Bano}^{5}$ ? Mais, en même temps, cela suscite une réflexion. Quoi qu'on pense du kitsch et des choses de ce genre, c'est un fait qu'au moins depuis deux siècles, et probablement à cause de la reproductibilité technique de tout, nous ne sommes plus naïfs, mais, quoi qu'on en dise, sentimentaux, c'est-à-dire incertains de notre goût, divisés entre l'idéal et le trivial.

Schiller l'avait très bien vu dans Sur la poésie naïve et sentimentale (I795-I796), qui est probablement la première réflexion explicite sur l'arc qui va du Camp au Postmoderne. Mais à l'époque il avait affaire aux romantiques de province dans les petits États allemands, et tout a changé lorsque le phénomène s'est universalisé, passant à des circuits culturels et industriels plus importants. C'est dans ce monde qu'au kitsch solitaire de Hölderlin qui dit que l'homme habite en poète se substituent Brian Jones habillé en rayures dans le Swinging London, Gina Lollobrigida, Victor Mature, Flash Gordon, et les revers monumentaux des costumes croisés de Gianni Agnelli.

Dans ce cadre du «tous libres", on verrait très bien Nietzsche: "Tous les noms de l'histoire, c'est moi ", écrivait-il à Burckhardt. Ou encore, comme l'écrivait Arbasino dans le fascicule du Super Eliogabalo, «Nietzsche, Adorno, Lacan, Totò ». Mon Camp. Ou mieux (passez-moi le Camp), mein Kampf, parce qu'en définitive, qu'y a-t-il de plus Camp qu'Hitler en armure de chevalier teutonique, comme le représentait un manifeste du Troisième Reich?

Laissons là les tamarri. Venons-en à une question philosophique centrale, qui est en définitive le noyau de toute notre enquête. Quand on dit que quelqu'un ne pleure pas vraiment, on suppose qu'il feint de pleurer, mentant aussi à lui-même. Quand on dit que quelqu'un ne rit pas vraiment, on sousentend ou bien qu'il rit pour de mauvaises raisons (et alors rira bien qui rira le dernier), ou bien qu'il feint de rire, qu'il rit d'un riz que l'on ne peut pas cuire. Comme le disait perfidement Edmondo De Amicis dans Cuore: un rire que les connaisseurs savent masquer, parce qu'ils utilisent des muscles différents de ceux que l'on utilise lorsqu'on rit de bon cœur. Mais voilà le nœud de l'affaire: personne n'a jamais douté que, contrairement au fait de pleurer, on puisse rire de bon cœur au sujet d'une fiction, et que quand on rit pour une fiction on rit vraiment.

C'est une considération que n'importe qui peut expérimenter sur luimême: le rire le plus beau est finalement celui qu'on a quand on nous raconte des blagues. Or si nous prenons ce point au sérieux, il faut en tirer beaucoup de conséquences. Qui contesterait qu'on ne rit pas vraiment pour des blagues? Et qui contesterait qu'elles racontent des faits véridiques? Ne pouvons-nous pas imaginer une scène dans laquelle Toto raconte une blague à Dédé, et où Toto, après avoir écouté la blague, dit : "Je te prie de me préciser si les faits racontés sont vrais, parce que je ne rirai qu'à cette condition. Je

4. Actrice italienne vulgaire et niaise (N.d.T.).

5. Chanteur populaire italien vulgaire et niais (N.d.T.). 
veux rire vraiment, et donc je ris seulement quand il s'agit de choses vraies». À coup sûr on peut l'imaginer, mais c'est vraiment une hypothèse tirée par les cheveux: disons que c'est aussi une espèce de blague.

C'est la thèse fondamentale. Les blagues sont l'exemple lumineux d'un rire authentique qui est complètement indépendant de la vérité ou de la fausseté des choses racontées. Évidemment, le monde est plein d'anecdotes amusantes, d'histoires drôles mais vraies, de gaffes de toute sorte, qui font rire et qui, en plus, sont vraies. Ou qui font rire exactement parce qu'elles sont vraies. Pourtant, lorsque quelqu'un dit: "Tu connais celle du pompier qui se dispute avec le rhinocéros? ", personne ne s'attend à un fait vrai qui se serait produit avec un pompier et un rhinocéros, parce qu'il est difficile d'imaginer un pompier qui se dispute vraiment avec un rhinocéros, comme ça l'est d'imaginer un renard qui dirait vraiment que les raisins sont trop verts.

L'absolu de la blague, sa sublime et très sérieuse indépendance de la réalité (pour dire que quelque chose n'est pas vrai, on dit "c'est une blague »), est confirmé aussi dans une autre circonstance: comme les mythes, les blagues n'ont pas d'auteurs. Songeons-y un instant: ne vous est-il jamais arrivé que quelqu'un vous accoste et vous dise: "Hier j'ai inventé cette blague "? Impossible ou, mieux, indicible. En effet, je n'exclus pas du tout que quelqu'un invente des blagues, car autrement on ne comprendrait pas comment ce genre a envahi la noosphère, le monde des pensées et des jeux sociaux. Le point est que, si quelqu'un invente une blague, il ne dit pas l'avoir inventée, et cela non pas par humilité, mais parce qu'autrement il ferait moins rire, sa blague serait moins l'expression d'un point de vue absolu et de l'esprit d'un peuple, pour parler un peu comme les romantiques et les nazis.

En ce sens, le narrateur de blagues se comporte vraiment comme un aède ou comme un rapsode. Ou, plus près de nous, comme un auteur médiéval, qui se garderait bien de mettre son nom sous son fabliau, parce qu'il sait que cela en diminuerait l'importance. Même chose pour les philosophes, qui se limitaient au commentaire et à la glose. Bref, le monde des signatures et des griffes, ou plus simplement de ce que plus banalement aujourd'hui nous attendons d'une œuvre, le fait que ce soit l'œuvre d'un auteur, était encore loin.

Le mythe, le fabliau médiéval, le commentaire philosophique ou théologique ne sont pas griffés, ils sont rigoureusement sans logo, la créativité de l'auteur, cette manie des siècles derniers, ne s'est pas encore imposée. Ensuite, les choses ont changé petit à petit, et nous avons assisté à un pullulement de poèmes, de romans, d'essais, et aussi de traités théologiques rigoureusement signés. Je crois que le droit d'auteur, et surtout les droits d'auteurs, a joué un certain rôle dans cette transformation.

Reste seulement deux sphères soustraites à la griffe: les prières, généralement anonymes, et, justement, les blagues. Les blagues «de Gino Bramieri » 
qui circulaient jadis devaient s'entendre comment «blagues récoltées par Gino Bramieri », et pas comme des «blagues créées par Gino Bramieri " 6 .

Mais s'il n'y a pas de satisfaction d'auteur à raconter des blagues, cela n'exclut pas qu'elles flattent notre narcissisme. Elles ne flattent pas notre ego d'auteur, mais d'exécutant. La responsabilité dans la manière dont on raconte une blague est en effet très grande: il n'y a pas blague, aussi bonne soit-elle, qui ne puisse être gâchée par un exécutant médiocre. Qui plus est, un exécutant de haut niveau trouvera toujours un moyen de rendre noble une blague médiocre.

Mais en quoi consiste cette excellence d'acteur? En un choix du moment où il faut raconter la blague, du temps qu'on y met, en la capacité d'imiter la voix des autres, la bonne mémoire. La mémoire est essentielle, il n'y a rien de plus déprimant que celui qui ne se rappelle pas comment finit la blague, ou qui se trompe sur la conclusion et conduit tout le monde à se demander "Mais qu'y a-t-il de drôle à cela ?» en le contemplant avec embarras.

Je ne m'étends pas sur cet aspect, parce qu'il y a beaucoup de blagues sur ce thème, mais je voudrais noter qu'une blague mal racontée peut carrément changer de sens et, au lieu de faire rire, faire pleurer. Cela dépend certes des circonstances, mais imaginons la noble scène de quelqu'un qui, sur son lit de mort, évoque avec un ami le bon temps, les vannes, les fêtes, les blagues. L'ami lui dit: «Tu te rappelles celle du pélican et de la Suisse?» Le mourant demande de la lui remémorer, l'ami rate la conclusion, le mourant fond en larmes.

Notons encore un autre point, dans cette ontologie de la blague, qui concerne ce qu'Umberto Eco a défini comme la coopération entre auteur et lecteur. Dans la blague, cette coopération est encore plus cruciale, et l'auditeur a un pouvoir de vie ou mort, celui de ruiner quand il le veut n'importe quelle blague. Il peut la tuer avant même que le narrateur ait prononcé un seul mot. Il lui suffit de dire, "Fais-nous rire» pour que personne ne rie. Il peut tuer la blague dans l'œuf: "Tu connais celle de l'ours et de la Polak?» — «Oui». Il peut la griller en cours de route, en se mettant à la railler et en faisant comprendre qu'il sait la fin. Il peut la griller à la fin, en riant lorsqu'il croit qu'elle est finie, alors qu'elle ne l'est pas encore, et en faisant comprendre qu'il rit seulement pour faire plaisir au narrateur. Et il peut la griller à la fin en ne riant pas, soit par bêtise (il n'a pas compris) soit par malice (il fait semblant de n'avoir pas compris).

S'il est vrai, comme je le crois, qu'on ne rit ni ne pleure jamais tout seul, il n'y a pas de blague qui ne puisse toucher un auditoire incompétent ou malveillant. Parce qu'en effet, en général, deux axiomes contradictoires sont vrais, terriblement vrais. Le premier dit: "On peut rire de tout ", et de fait il y a occasion à rire de tout, il n'y a pas de tragédie ou d'enterrement

6. Comique italien vulgaire et niais, passeur de Barzelettes, petites blagues ou blagounettes (N.d.T.). 
qui n'aie pas un côté comique. Mais le deuxième dit: «Il n'y a pas de quoi rire. » Et vraiment, c'est exactement cela. Comme dirait Pascal en parlant de la vie, aussi belle qu'ait été la comédie, le final est toujours tragique.

Récapitulons notre ontologie de la blague: I) La beauté de la blague ne réside pas dans la réalité de la référence: une blague ne fait pas rire plus si ce dont elle parle est vrai; au contraire, si une chose vraie mais ridicule apparaît incroyable, on dit: "C'est de la blague». 2) La beauté de la blague ne réside pas dans le prestige de l'auteur. Si une blague attribuée à Goethe ou à Michel-Ange ne fait pas rire, elle ne fait pas rire, et c'est tout; et cela indépendamment du fait que, comme nous l'avons vu, la stratégie qui prévaut est de la rendre anonyme (ou, plus exactement sans auteur). 3) La beauté de la blague réside tout entière dans la construction, dans le choix des temps, dans l'exécution du récit, c'est-à-dire dans le montage et dans la performance. 4) Toutefois, il n'y a pas de blague, aussi bonne soit-elle, qui ne puisse rater à cause d'un auditoire bête ou taquin.

On remarquera que ce que j'ai dit de la blague est valable pour beaucoup d'autres formes d'art et, après tout, ce que Kant a dit du beau dans la Critique du jugement s'applique parfaitement aux blagues, y compris le fait que le jugement esthétique n'est pas contraignant et que, face à quelque œuvre d'art que ce soit, comme face à toute blague, j'ai toujours le droit de dire "elle ne me plaît pas»; personne au monde n'aura autorité pour me dire que je me trompe, que je n'ai pas le droit de ne pas apprécier cette œuvre d'art ou cette blague. (On se venge en disant que je n'ai pas de goût ou que je manque d'esprit.)

L'analogie entre les blagues et l'art a une seule exception, l'avant-garde, qui contredit la règle (2), puisque dans l'avant-garde le prestige de l'auteur compte, et même dans certains cas il n'y a que cela qui compte, car l'on n'a pas affaire à de l'avant-garde si l'on montre que le soi-disant auteur de l'œuvre n'était pas le précurseur des sacs griffés, des cravates signées, de l'invasion des logos.

C'est le moment de conclure nos variations sur le rire et le pleurer vraiment. Je voudrais suggérer une réflexion finale. Probablement, « rire ou pleurer vraiment ", le paradoxe de la fiction, repose dès le départ sur un sophisme qui consiste à assumer que la seule activité des êtres humains est de juger, de décider si des propositions sont vraies ou fausses. Et il est clair que si l'on adopte ce cadre, la question se pose de savoir si lorsque que je pleure pour Anna Karénine je pleure vraiment.

Mais dans la vie, nous ne passons pas tout notre temps à juger. Si l'on y prête attention, nous faisons un tas d'autres choses: nous donnons et exécutons des ordres, nous offrons et acceptons des excuses, formulons des prières et des saluts, lançons des insultes, donnons des noms et des titres, faisons des promesses et signons des contrats, lisons des livres et écoutons des concerts. À la limite, comme le fameux personnage de Nani Moretti, nous faisons des choses et voyons des personnes. Dans toutes ces activités, le jeu 
de dénoter, d'indiquer quelque chose et de décider si elle existe ou pas, et si les mots employés pour les définitions sont corrects ou incorrects, est de loin une activité marginale par rapport à mille autres pratiques plus répandues.

En dépit du fait que - et peut-être parce que - faire des théories est l'activité favorite des professeurs, notre jeu fondamental dans le monde n'est pas de formuler des jugements vrais et motivés, c'est-à-dire des jugements scientifiques. C'est une activité en réalité très importante, mais bien plus rare qu'on ne le croit, et pratiquée seulement en de rares occasions par ces mêmes scientifiques. Noli iudicare, plus qu'un précepte moral, est la description de ce que ce qui s'impose dans la majorité des cas. Non seulement juger est une activité rare, une île au sein d'un archipel d'autres pratiques, mais dénoter, indiquer quelque chose qui accompagne cette activité de jugement est vraiment une chose très rare, qui ne saisit pas les aspects importants de notre expérience, ces aspects que nous poussent à voir un film ou un tableau, à écouter de la musique, à lire un roman. Dans ces activités, ce qui domine est quelque chose comme simplement montrer. À l'origine, il y a des situations comme celle des deux personnes qui sont en train de faire une promenade et trouvent un lézard sur le sentier. L'un dit à l'autre «Regarde! », l'autre regarde, et souvent ne dit rien. La même chose se passe avec les couchers du soleil, et avec quantité d'autres choses, et parfois (c'est un grand moment) avec les tableaux des musées, à condition que notre voisin ne se sente pas en devoir d'accompagner le «regarde!» par quelque commentaire inutile et souvent inopportun.

L'équivalent du montrer est le regarder qui, là aussi, peut très bien arriver sans être accompagné d'un jugement quelconque. Bref, il n'est pas vrai que les intuitions sans concept et sans jugement soient aveugles. Elles voient très bien. Comme dans la scène de la mort de Bergotte dans la Recherche, lequel va voir une exposition de Vermeer, trouve le tableau qu'il aime le plus, celui dans lequel est représenté un petit pan de mur jaune, s'assied heureux face à ce tableau, sent que les forces vont lui manquer, et formule un jugement qui n'a pourtant rien à voir avec le tableau et avec son bonheur: "Voilà, maintenant je meurs.»

Une autre activité souvent blâmée par les philosophes, mais dont la vie est remplie, est l'exemplification. On se rappelle que Socrate demandait quelle est l'essence du musicien, et lorsqu'on lui donnait des exemples de musiques il se mettait en colère parce que ce n'était pas l'essence: «Je t'ai demandé ce que c'est qu'un musicien, et tu m'as répondu en mentionnant des musiques! »

Mais sommes-nous vraiment sûrs qu'il ne faille pas agir ainsi? En effet, exemplifier est l'une des choses les plus communes dans notre existence, et la preuve en est donnée exactement par le fait que les interlocuteurs de Socrate trouvaient très naturel de lui répondre par des exemples. C'est pour une raison très simple: nous ne disposons pas du tout (ou du moins ne disposons que très rarement) des essences. Ce que nous avons, ce sont plutôt des analogies, des ressemblances, des à-peu-près, toutes choses qui ne se rendent pas 
par des exemples. Donc, il n'y a rien de mal, si n'avons pas l'essence (et je répète que l'avoir est difficile), à répondre à quelqu'un qui nous demande "Qu'est-ce qu'un musicien?» — "c'est un type comme Mozart, comme Jovanotti, comme Dylan».

L'exemplification est l'une des activités dominantes de l'art. Et ce que Tolstoï, par exemple, a voulu nous dire avec la mort d'Anna Karénine n'est pas simplement ce qui peut résulter du jugement: "Celui qui va contre les conventions de son époque, qu'elles soient justes ou iniques, finit mal.» Il aurait suffi d'énoncer le jugement en deux lignes. S'il en a écrit beaucoup plus, c'est justement pour exemplifier, et que l'exemplification soit réussie est montré par le fait que, face à la mort d'Anna Karénine, on peut pleurer vraiment.

Mais alors, pourquoi la mort d'Anna Karénine est-elle survenue, pourquoi fait-elle pleurer vraiment, bien qu'elle ne soit pas vraie? Simplement parce que dans des choses comme le montrer, le regarder, et surtout le comment exemplifier, la catégorie dominante n'est pas celle du vrai/faux, comme dans les jugements, mais plutôt un couple différent, celle de «heureux/malheureux ", qui a été introduite au siècle dernier par le philosophe anglais John L. Austin.

Austin a porté l'attention sur un usage particulier du langage qu'il appelait "performatif ", dans lequel les mots ne servent pas à dire si une chose est vraie ou fausse, ou à poser une question, mais créent une chose qui n'existait pas auparavant. L'exemple classique est celui du mariage: en disant «oui », je ne décris pas un mariage, mais je le fais. Or, avec les performatifs, on ne peut pas faire valoir la règle du "vrai/faux", exactement parce que cette règle suppose qu'il y ait une proposition qui s'adapte à la chose, alors qu'ici la proposition construit la chose. La catégorie dominante ici sera différente: c'est la distinction "réussi/raté ». Pour comprendre ce dont il s'agit, pensons aux blagues, où, comme on l'a vu, le fait qu'elles soient vraies ou fausses a peu d'importance, et où ce qui compte le plus est qu'elles soient bien racontées dans le contexte approprié. S'il y a une erreur dans la performance, ou dans le contexte ( on ne parle pas de corde dans la maison d'un pendu»), alors la blague, pour autant qu'on voulait rendre heureux, est malheureuse, ratée, pas réussie. Mais si la blague ou la tragédie est, pour ainsi dire, performée de façon juste et dans le contexte approprié, alors elle est heureuse et nous fera rire vraiment, exactement comment la tragédie nous fera pleurer vraiment.

Il est temps d'en finir. Y a-t-il une morale à tout ça ? Peut-être. Je crois avoir montré suffisamment que, pour rire et pleurer vraiment, il n'est pas nécessaire que les choses qui nous font rire ou pleurer soient vraies, et qu'au contraire, dans la plupart des cas, le fait que les choses soient vraies ne renforce pas le sentiment, mais le transforme: le rire devient pleur, les pleurs deviennent rires et, ne l'oublions pas, ce qui finit par dominer est l'ennui, 
«ce monstre délicat», comme disait Baudelaire dans la dédicace des Fleurs du mal.

En effet, alors qu'on ne se lasse pas de voir la tragédie du Roi Lear, d'écouter les malheurs de notre prochain et souvent aussi de penser aux nôtres, les pleurs cèdent la place à l'impatience, à la gêne, à l'ennui, et finalement même à la colère. "Tout passe, tout lasse, tout casse, tout se remplace»: ce dicton infaillible n'a pas été forgé pour l'art mais pour la vie. Il n'y a alors pas lieu de s'étonner du fait que, pour rire ou pleurer vraiment, on recoure à ce qu'on appelle confusément la «fiction".

On a même relevé ce qu'on pourrait appeler le «syndrome des sentiments fictifs", qui frappe les personnes incapables de s'émouvoir pour les malheurs vrais de leurs prochains, mais qui au contraire compatissent avec Werther ou s'émeuvent du destin tragique d'Oliver Twist. "Mais quoi, ce sont des fous, et je ne serais pas moins fou de me régler sur leur exemple», dirait probablement Descartes; toutefois, étant donné que nous avons évoqué la dédicace des Fleurs $d u$ mal, peut-être serait-il plus approprié de s'adresser à eux comme le fait Baudelaire: «Hypocrite lecteur, mon semblable, mon frère ${ }^{7}$.

7. À une exception près, le contenu de cet essai est sorti dans «Torino 7 ", supplément local de La Stampa, dans la rubrique «Metaphysical Club» que je tiens sous le pseudonyme de Perry Mail. Un remerciement spécial va à mon frère, Gabriele Ferraris, qui a accepté ces articles dans le supplément qu'il dirige, et autorisé leur réédition. Tous ces motifs pragmatiques (parce qu'après tout mon frère est mon supérieur, au moins lorsque je porte le masque de Perry Mail) et sentimental (parce qu'avant tout mon frère est mon frère) font que Pleurer et rire pour de vrai lui est affectueusement dédié. 